\title{
Evaluation of a nonlinear reef parametrisation for steady flows
}

\author{
Lance Bode* Luciano B. Mason ${ }^{\dagger}$
}

(Received 8 November 2004; revised 14 September 2005)

\begin{abstract}
Modelling ocean circulation in regions of high topographic complexity, notably around groups of reefs and islands, makes large demands on spatial resolution. This problem has largely been overcome by a parametrisation scheme in which the dynamics associated with flow around unresolved reefs and islands are represented by modified momentum equations on a relatively coarse grid. However, the performance of this scheme deteriorates at high velocities, due to the increasing importance of flow separation and eddy formation, processes that are excluded in the original scheme. We extend the earlier model to include a parametrisation of the nonlinear advective terms, and test the performance of the modified scheme in the case of steady flow.
\end{abstract}

*School of Mathematical and Physical Sciences, James Cook University, Townsville, Australia. mailto:Lance.Bode@jcu.edu.au

${ }^{\dagger}$ School of Engineering, James Cook University, Townsville, Australia. Current address: Australian Maritime College, Maritime Way, Newnham, Australia

See http://anziamj.austms.org.au/V46/CTAC2004/Bode for this article, (c) Austral. Mathematical Soc. 2005. Published October 8, 2005. ISSN 1446-8735 


\section{Contents}

1 Introduction

C1018

2 Original reef model

C1019

3 Parametrisation of advective terms

C1023

4 Results

C1028

5 Discussion

C1030

References

C1033

\section{Introduction}

The spatial complexity of regions such as the Great Barrier Reef (GBR) poses major problems for ocean circulation modelling. As an illustration, Figure 1 shows a sub-region of the GBR, specifically the Pompey Complex offshore from Mackay. Reef shapes and sizes are such that explicit resolution of reef outlines is in general computationally infeasible, at least for models at regional and larger scales. Given that time steps for 2D models typically vary with spatial resolution as $\Delta t \sim(\Delta s)^{-3}$, the CPU cost implications are profound. This is especially so if such models are to be used in ecological applications, where integrations over months and years can be required. Such considerations led us to develop a relatively simple reef parametrisation scheme [2], which has been successfully applied to tidal and other circulation modelling in the GBR $[3,8]$.

In the original scheme [2] the effects of the nonlinear advective terms were not considered. However, physical and scaling considerations suggest that omitting them is not always justified, as evidenced by satellite imagery in 


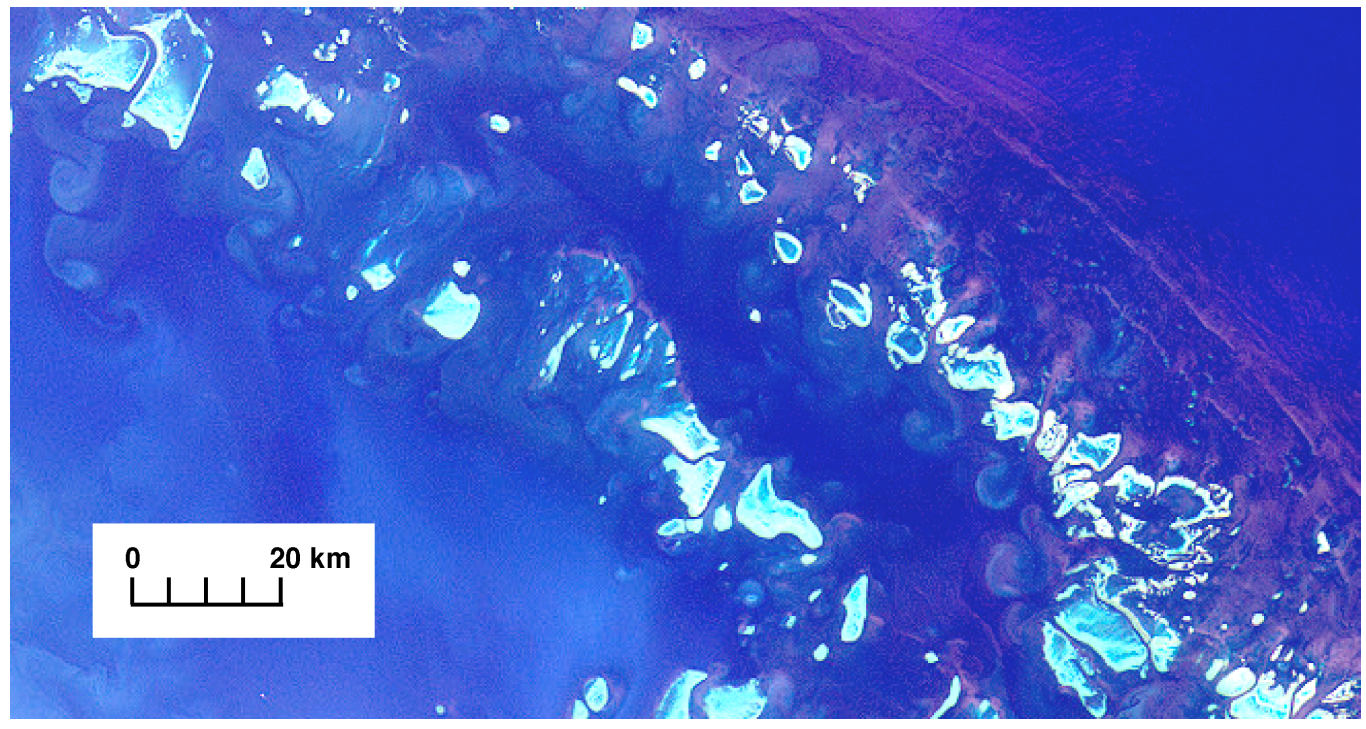

Figure 1: Satellite image of the Southern GBR; sediment and biological activity reveal eddies and other features of the tidally-dominated circulation.

areas of large tidal currents in the GBR (Figure 1). This shows pronounced jets and eddies behind reefs, evidence of the importance of the advective terms. Here we describe an extension of the scheme in [2] to incorporate a parametrisation of the nonlinear advective terms. Section 2 briefly discusses the original scheme. In Section 3 we consider how to extend this model to include the advective terms numerically. The performance of the modified scheme for steady flows through reefal regions is assessed in Section 4 using an idealised numerical test bed.

\section{Original reef model}

Bode et al. [2] showed that, within the framework of a two-dimensional finite difference (FD) model, reefs in each grid square are effectively represented by 


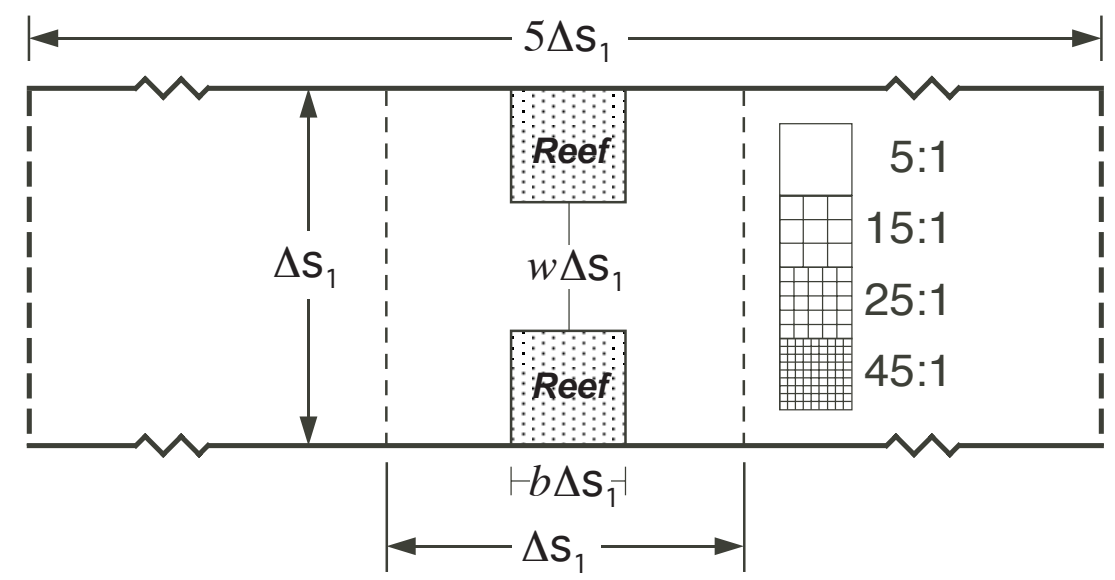

FiguRE 2: Schematic of idealised reef geometry, showing parameters $w$ and $b$ for $x$-directed (left to right) flow. This is also the numerical testbed used in the present work, with a width of 5 nautical miles $(1 \mathrm{~nm}=1.8532 \mathrm{~km})$, and an aspect ratio of $5: 1$; the depth is a constant $30 \mathrm{~m}$. The figure shows the coarsest grid scale, $\Delta s_{1}=5 \mathrm{~nm}$, used in the parametrised model, along with successively higher levels of grid refinement, typical of those used in the numerical experiments, and which all resolve the constriction explicitly.

a pair of parameters for each horizontal coordinate. These are termed the 'gap fraction' $w$ and 'width fraction' $b$, as depicted in Figure 2. The scheme was initially developed by considering quasi-1D flow through orifices with linear bottom friction. This was then made increasingly more realistic by:

1. incorporating 2D (or 'end') effects from an analytical solution of Huthnance [7];

2. extending the formulation to accommodate quadratic bottom friction; and

3. also allowing flow to occur over the tops of reefs as well as through the gaps between them. 
With purely linear dynamics, the momentum equations for the various component sections of each grid square are combined [2] to show that $U$, the overall transport (or depth-integrated velocity) for $x$-directed flow depends linearly on the imposed pressure gradient via an impedance formula, $U=$ $i g h(\partial \eta / \partial x) / \sigma_{e}$. Here $\eta$ is sea surface elevation, $h(x, y)$ is ambient depth and $g$ is gravitational acceleration. This formula has clear electrical circuit analogies. Locally, impedance is defined by $\sigma=\omega-i r / h$, where $r$ is a linear friction factor and $\omega$ is the angular frequency of the forcing (for example, tidal); all dependent variables are assumed to be proportional to $e^{i \omega t}$. However within each grid square when reef elements are incorporated, the effective impedance $\sigma_{e}$ is due to the impedances of the reef and non-reef (or continental shelf) components acting in series: $\sigma_{e}=b \sigma_{r}+(1-b) \sigma_{s}$. The impedance for the reef component (due to flow both over the reef crest and through interreef gaps) is given by an impedance in parallel: $\sigma_{r}^{-1}=\left[w / \sigma_{s}+d(1-w) / \sigma_{c}\right]$, where $d=h_{c} / h_{s}$ is the ratio of water depths for the reef crest and shelf.

Although the impedance approach provides useful conceptual insights, its application is limited to simple analytically tractable cases. Of more value to practical numerical modelling in areas such as the GBR is the fact that this approach also yields equivalent momentum equations. Further, the linear model can be generalised to incorporate the more realistic case of quadratic bottom friction. The net result in this situation is an effective horizontal momentum equation, which for $x$-directed flow is written as

$$
\alpha \frac{\partial U}{\partial t}=-g H \frac{\partial \eta}{\partial x}-\beta \frac{\lambda|\mathbf{U}| U}{H^{2}},
$$

where $\mathbf{U}=(U, V)$ is the transport vector, $H=h+\eta$ is total water depth, and $\lambda$ is a quadratic bottom friction coefficient. At this stage the nonlinear advective terms have been excluded from consideration, whereas Coriolis terms are omitted for simplicity. Importantly, the terms $\alpha$ and $\beta$ that act to modify the effective pressure gradient and bottom friction across a given grid square are expressed in terms of the reef parameters $w$ and $b$, and thus are fixed factors that depend on reef geometry in the grid square under consideration. 
For example, in the most basic quadratic friction model presented in [2], a case in which no flow is permitted across reef crests, $\alpha=(1-b)+b / w+\Phi$ and $\beta=(1-b)+b / w^{2}+1.56 w^{-0.56} \Phi$, where $\Phi(w)$ is the term associated with $2 \mathrm{D}$ end effects, as determined by Huthnance [7]. Further details and a number of generalisations of this model can be found in the original paper.

Results from this parametrised scheme compare closely with those from high-resolution explicit simulations that are orders of magnitude more expensive computationally [2]. The schemes were assessed in a numerical testbed (Figure 2), a constricted channel in which flow is driven by pressure (sea level) differences at the ends. The dimensions of the channel $(25 \times 5 \mathrm{~nm}$; $1 \mathrm{~nm}=1.8532 \mathrm{~km}$ ) are typical of those found across sections of the GBR. The coarsest resolution uses a grid spacing of $\Delta s_{1}=5 \mathrm{~nm}$ (or $1: 1$ resolution), at which scale the "reef" in Figure 2 is not resolved by the grid, and hence must be parametrised by setting appropriate $w$ and $b$ values. In all other simulations, the reef is explicitly resolved, using successive levels of spatial refinement. In present work these are 5, 25, 75 and $125: 1$. For the final case, the grid length is reduced in size to $\Delta s_{125}=\Delta s_{1} / 125 \approx 74 \mathrm{~m}$. The model domain now contains around $8 \times 10^{4}$ grid points, versus just 6 for the coarsest (parametrised) model.

When applied to circulation modelling in the GBR, the scheme is shown to be accurate and flexible: once the parameters for each grid square are established, the equations of motion can be solved by any standard FD scheme. In our case this is the implicit model described in [1]. The underlying problem for application of the scheme is to estimate the effective $w$ and $b$ for each grid square in each horizontal direction. Briefly, this involves using high resolution digitised outlines of reefs, islands and bathymetry. Within each FD square, this information is incorporated into a high resolution channel flow setup, similar to that of Figure 2. Steady-state flow is then computed for a given pressure gradient, and from the results and the additional constraint of reef area, $w$ and $b$ values are determined. This procedure is computationally intensive but has been automated, and needs to be performed just once for 
a given location and grid setup.

Despite the computational advantages and economy of such an approach, its use does not appear to have gained significant acceptance over traditional methods. For example, a recent $3 \mathrm{D}$ model on a $1 \mathrm{~km}$ grid of the complex region around Singapore [11] employs "manual node-to-node editing" to treat subgrid-scale features such as causeways, narrow channels and small islands. Our approach is designed to parametrise this type of geometric detail in a dynamically consistent and accurate manner. Studies that have attempted to parametrise subgrid-scale geometry are the original analytical models of Huthnance [7], and a relatively simple approach by Metzger and Hurlburt [9] that used modified frictional dynamics to incorporate the effects of flow over unresolved sills in an ocean general circulation model. Recent discussions of the importance of adequate spatial resolution in ocean models can be found in [6] and [12]. A related but simpler procedure has been successfully developed for large-scale spectral wind-wave modelling [4].

\section{Parametrisation of advective terms}

In the above model, we assumed that advective terms can be ignored. The validity of this assumption can be assessed by scaling analysis or by direct numerical solution; here we use the latter approach. The dynamics are governed by the continuity and momentum ( $x$ only, for brevity) equations. In transport form these are expressed as

$$
\begin{gathered}
\frac{\partial \eta}{\partial t}+\frac{\partial U}{\partial x}+\frac{\partial V}{\partial y}=0 \\
\alpha \frac{\partial U}{\partial t}+\frac{\partial}{\partial x}\left(\frac{U^{2}}{H}\right)+\frac{\partial}{\partial y}\left(\frac{U V}{H}\right)-f V=-g H \frac{\partial \eta}{\partial x}-\beta \frac{\lambda|\mathbf{U}| U}{H^{2}}
\end{gathered}
$$

where $\alpha$ and $\beta$ arise from the existing reef model of Section $2, f$ is the Coriolis parameter, and other variables have been already defined. 

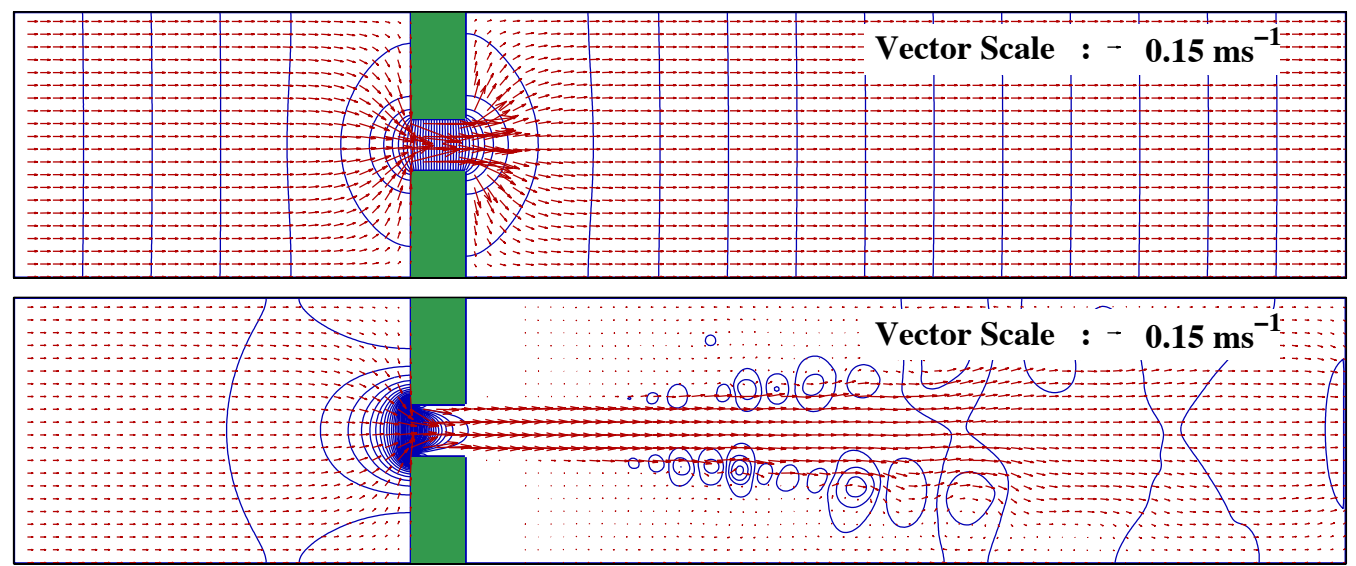

Figure 3: Numerical solutions for a constant head difference of $\pm 5 \mathrm{~mm}$ at the channel ends, using the highest grid scale of $1 / 125$ of the parametrised resolution. Equi-spaced surface elevation contours $(\Delta \eta=0.2 \mathrm{~mm})$ and velocity vectors are shown: (i) without advective terms, top panel; (ii) with advective terms included, bottom panel. Vectors are shown at every 7 th grid point to aid visibility. (Note: magnify PDF figures to show high-level detail.) The reef constriction is offset to reduce downstream boundary effects.

In order to demonstrate the importance of the advective terms, steady flow is computed in the testbed channel of Figure 2. This is done by imposing what might be considered moderate forcing in the context of flows through the GBR: constant elevations of $\eta= \pm 5 \mathrm{~mm}$ at the ends of the channel, or a total head loss of $10 \mathrm{~mm}$ over its $25 \mathrm{~nm}$ length. Note also that no flow is allowed over the reef crest in the present case. The model is integrated to steady-state from an initial condition of quiescence. The grid resolution employed, $\Delta s_{125} \approx 74 \mathrm{~m}$, is the finest of any used in the present study. This is $1 / 125$ of the coarsest possible grid size, $\Delta s_{1}=5 \mathrm{~nm}$.

Figure 3 shows computed velocities and sea levels. The plots correspond to: (i) no advective terms (that is, the original model [2]); and (ii) with the advective terms included. Respective maximum velocities, averaged across 
the reef gap midline, are 0.60 and $0.33 \mathrm{~m} \mathrm{~s}^{-1}$. Pressure contours and velocities in (i) are approximately symmetric about the gap, so that this basically corresponds to frictionally-modified ideal flow.

Inclusion of the advective terms is seen in (ii) to reduce the transport markedly, at least for this fairly restricted geometry, but it also results in pronounced changes to the nature of the flow. This now exhibits the characteristic features of a slowly diverging jet. Almost all the pressure variations occur upstream of the efflux region, so that additional form drag would be expected.

Bernoulli arguments produce sufficiently accurate flow estimates if required. Assuming a non-divergent jet downstream of the gap, and making sensible (conservative) approximations for the influence of quadratic bottom friction within the channel, we obtain

$$
u_{\text {gap }} \approx\left[\frac{2 g \Delta \eta}{1-w^{2}+2 \lambda w L / h}\right]^{1 / 2},
$$

where $\Delta \eta$ is the total head loss along the channel of total length $L$. Ignoring friction $(\lambda=0)$ gives $u_{\text {gap }}=0.45 \mathrm{~m} \mathrm{~s}^{-1}$, whereas the frictional approximation of $0.31 \mathrm{~m} \mathrm{~s}^{-1}$ is much closer to the value of $0.33 \mathrm{~m} \mathrm{~s}^{-1}$ computed above.

Numerical approach: we aim to provide a numerical scheme to represent advective processes with acceptable accuracy for coarse-scale (parametrised) models of flow around reefs. To this end it is instructive to recast the advective terms in equation (3): by subtracting $U / H$ times the continuity equation (2) from the advective terms we obtain

$$
\frac{\partial}{\partial x}\left(\frac{U^{2}}{H}\right)+\frac{\partial}{\partial y}\left(\frac{U V}{H}\right)=\left[\frac{U}{H} \frac{\partial U}{\partial x}+\frac{V}{H} \frac{\partial U}{\partial y}\right]-\frac{U}{H}\left[\frac{\partial \eta}{\partial t}+\frac{U}{H} \frac{\partial H}{\partial x}+\frac{V}{H} \frac{\partial H}{\partial y}\right] .
$$

Since $(U, V) / H$ is depth-averaged velocity, the individual bracketed terms in (5) have two quite distinct interpretations. The first bracket has the usual 
form of the advection of $U$ by the velocity field. The second is associated with changes in water depth, or equivalently could arise from changes in the channel cross-section. Importantly, the second bracket has a geometric origin: apart from small variations associated with changes in water level, the spatial derivatives are determined by the local geometry, and are not associated with variations in the prognostic variables $U$ and $V$.

The point of writing the advective terms in this form becomes clearer when we now consider our constant depth channel from two distinct viewpoints, depending on the grid resolution that applies. The first corresponds to a high resolution grid, such as that in Figure 3, where all bathymetric detail is explicitly resolved. In this case all three terms in the second bracket are effectively zero at each active computational point, and the advective processes are thus associated with explicitly resolved spatial gradients in the transport field, as given by the first bracket. These effects will be most pronounced in the immediate neighbourhood of the reef constriction.

Importantly, this situation is reversed for a parametrised reef model, where the geometric detail around the constriction cannot be resolved by the grid. In this case, using the coarsest grid scale $\left(\Delta s=\Delta s_{1}\right)$, both $\partial U / \partial x$ and $V$ are sensibly zero, and thus the first bracket can be ignored. Therefore the advective accelerations, when averaged over any grid square, need to be given accurately by an appropriate numerical representation of the second bracket in equation (5). In other words, at the parametrised scale, the reef constriction in each grid square needs to be represented by bathymetric variations. We recognise here that these terms have essentially the same form as the advective terms for flow in a channel of varying cross-section [5]. In the case of our constant-depth constricted channel, it is actually width rather than depth that is changing at the subgrid scale. Therefore, guided by this channel flow analogy, we replace $H$ by an effective depth, $H^{\prime}=w H$, in order to maintain the appropriate cross-sectional area, $H(w \Delta s)=H^{\prime} \Delta s$, across the constriction.

However, modifying the definition of $H$ in a numerical scheme is not 
sufficient to obtain accurate parametrised solutions. Further consideration of the flow in Figure 3 shows that, at the coarse scale, the dominant advective term in equation (5) is $(U / H) \partial U / \partial x$. Assuming the basic flow is from left to right $(U>0)$, then if we encounter a region of decreasing depth (or crosssectional area) over one grid square, this term is negative and the net effect in the momentum equation is resistive, thus reducing the transport in the channel for a given pressure gradient. If on the downstream side the flow fanned out as in Figure 3(i), then by the same argument, the opposite effect would apply, meaning that this term would have no net effect on the flow. This is counter to reality and to the nature of the flow revealed in Figure 3(ii). The flow downstream of the constriction has separated and resembles a jet, with a cross-sectional area that remains approximately constant. That is, $(U / H) \partial U / \partial x$ is effectively zero downstream of the constriction, and hence the contribution of the advective terms to the momentum balance must be determined by upstream processes. This physical situation has to be reflected by its numerical representation, and therefore requires a special treatment.

In our numerical reef model, we compute all advective terms in the form given by equation (5). Following the arguments given in the paragraph above, we compute the second bracket of (5) in an upstream sense, with $H$ replaced by the effective depth, $H^{\prime}=w H$. If the value is negative, this means that the flow is entering a constriction, regardless of the sign of $U$, and hence corresponds to flow retardation. In such a case this upstream value of the derivative of $H^{\prime}$ is used in calculating the second bracket of (5). By contrast, if the term is positive, it is set to zero; otherwise it would spuriously accelerate the flow in a grid square downstream of the constriction. Clearly this approach is a crude representation of reality: we have ignored the details of the flow downstream of the constriction, where some small proportion of the pressure field will be recovered. Although it might be possible to "tune" the scheme to give improved results over the current model, this temptation has been avoided. The present approach is simple to implement numerically, and appears to give quite acceptable results, as described in the following section. 
Note that our approach does not result in a low order upwind scheme, which would be highly diffusive [10]. Here, the term that parametrises the form drag associated with the constriction, and hence leads to a reduction in the transport through it, is computed in an upstream sense, because this mimics the physical processes that apply. This derivative term is geometric. It does not involve differences of prognostic variables, and hence does not introduce the problems that are normally associated with upwinding. Note also that if centred differences were used for an isolated reef element, the value computed for this term would be zero, and hence such a scheme would not "see" the constriction. In our model, the first bracketed term is still computed by the standard FD approach described in [1] — its effects will be negligible in the case of a parametrised reef element, as discussed above.

\section{Results}

The modified reef parametrisation model is assessed by using the same numerical testbed as in [2] (Figure 2) for the case of steady flow only; extensions to tidal forcing will be considered elsewhere. Results are compared against those obtained by explicit resolution of the constriction in the channel. Unlike [2], only one geometry is discussed here $(w=b=0.2)$, but this is a demanding case. It is also representative of the values that would apply in modelling a highly constricted reefal area (such as in Figure 1) at a relatively coarse spatial scale, in this case $\Delta s_{1}=5 \mathrm{~nm}$. Progressive grid refinements as large as 125:1 are used in the high resolution experiments. In addition, a number of different pressure gradients are imposed along the channel, in order to produce a wide range of velocities through the constriction.

Figure 4 shows the effect of grid resolution on the average transport computed in the channel. The leftmost points correspond to the $1: 1$ or parametrised grid. For the various cases in which the constriction is explicitly resolved, the computed transports are higher than the parametrised 


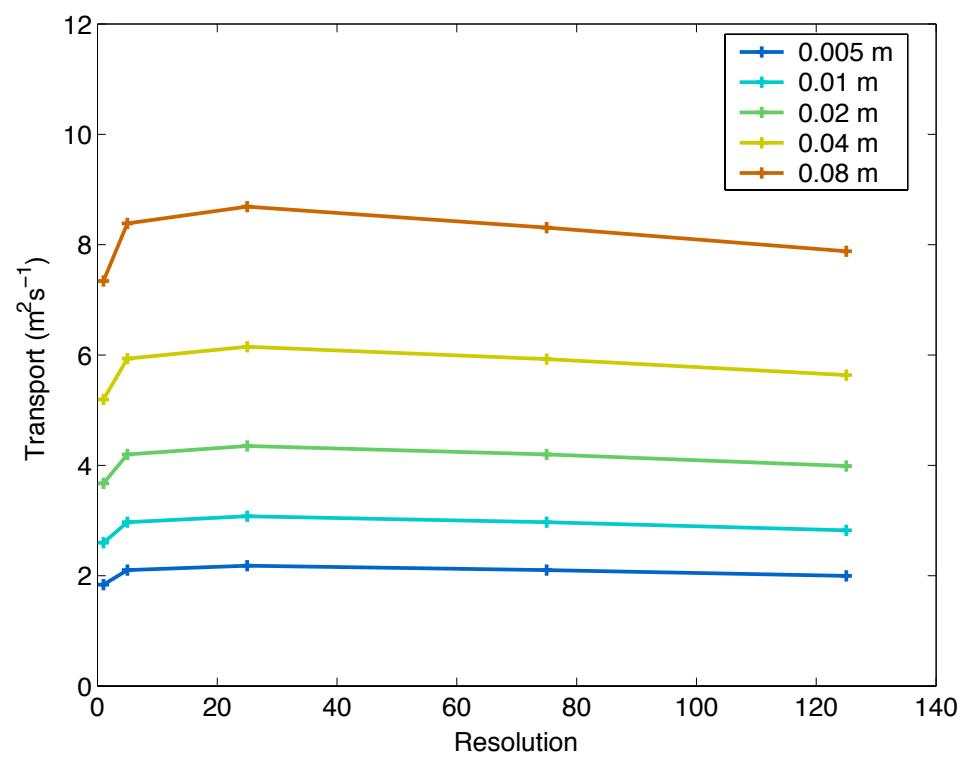

FiguRE 4: Transport across the constriction as a function of grid resolution, for various imposed pressure gradients along the channel. The pressure gradient is shown by the head difference along the channel: for example, $0.01 \mathrm{~m}$ corresponds to $\Delta \eta= \pm 5 \mathrm{~mm}$ at the channel ends. Resolution is shown as a fraction of the parametrised grid size, $\Delta s_{1}=5 \mathrm{~nm}$; thus ' 125 ' represents the finest grid used, $\Delta s_{125}=\Delta s_{1} / 125$, and corresponds to the flow shown in Figure 3(ii).

result but tend to decrease with grid size. This is not unexpected because the assumption of no flow divergence downstream of the reef constriction is not completely valid. Nevertheless, it is satisfying to note that the results obtained from the parametrised model are not obviously inferior to those obtained by high resolution computations that are 4 to 5 orders of magnitude more expensive computationally.

Further information on the accuracy of the new scheme is obtained by comparing these solutions with those obtained from the original scheme [2], 
that is, when the advective terms are eliminated. These results are given in Figure 5. Firstly, note that the parametrised model is extremely accurate in the non-advective case, as reported in [2]. Results for the case when advection is parametrised are not as accurate, but are still most acceptable. However, it is clear that for steady (or slowly varying) flows, at least with highly constricted geometries, it is unsafe to ignore the advective terms. On a log-log scale, the two steady-state families of curves are parallel, with exponent $\frac{1}{2}$, as could be expected from dimensional considerations: this is the basis of equation (4). Explicit resolution of flow details, even with extremely fine grids, is obviously difficult in cases where the flow is separating and eddying in the lee of these bluff "reefs", as should be apparent from Figure 3. However, the parametrised model, which is concerned only with calculating gross measures of the flow, averaged over a coarse grid square, would appear to perform almost equally as well as the far more costly explicitly-resolved simulations.

\section{Discussion}

An extension has been presented to the reef parametrisation scheme in [2] to cover situations where strong flows through reef gaps can cause downstream separation and eddying. When large pressure gradients and pronounced restrictions in the flow geometry combine to produce high velocities, the advective terms cannot be ignored in the momentum balance. Indeed they are often a dominant component, and hence need to be incorporated faithfully in numerical models. Moreover, the geometric complexity of such regions means that a parametrised approach to reef modelling may be required.

Here we approximate the form drag via a formulation that takes account of the geometric effects that tend to dominate the computation of the advective terms when subgrid-scale reef details have to be parametrised. The method is based on a simple heuristic approach, although it is motivated 


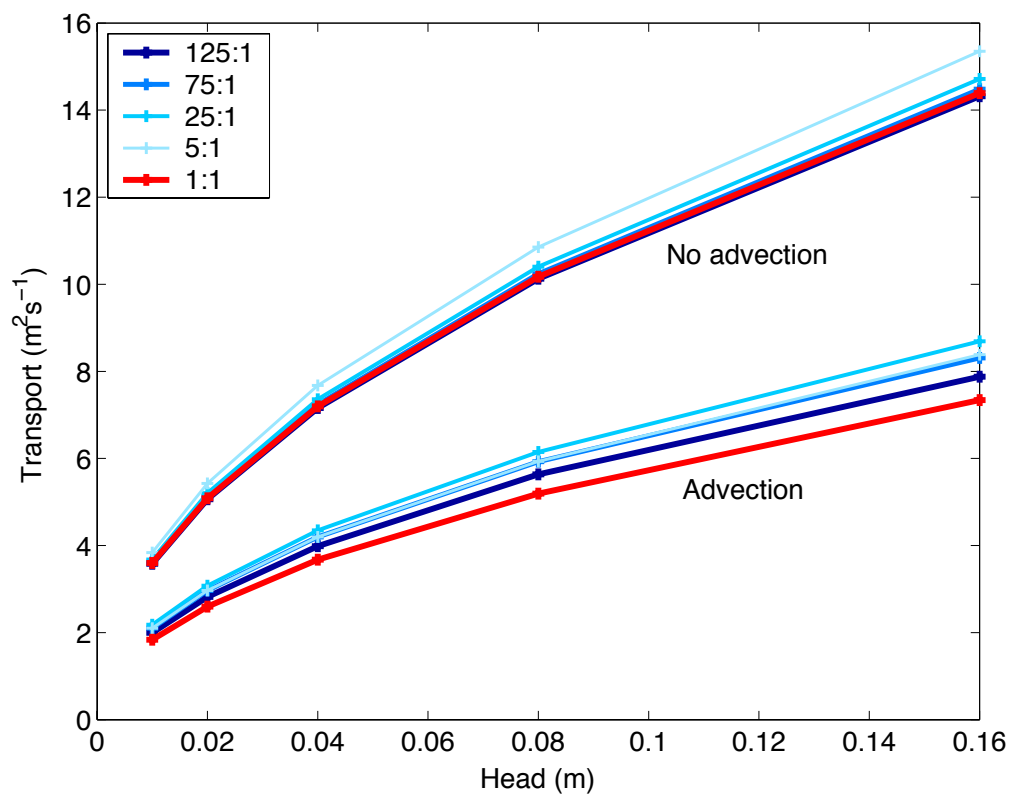

FiguRE 5: Transport across the constriction as a function of imposed pressure gradient with a range of grid resolutions, for both advective and nonadvective cases. Resolution is given as a fraction of the parametrised grid size, $\Delta s_{1}=5 \mathrm{~nm} ; 125: 1$ corresponds to the finest grid used, $\Delta s_{125}=\Delta s_{1} / 125$. The pressure gradient is shown by the head difference along the channel: for example, $0.01 \mathrm{~m}$ corresponds to $\Delta \eta= \pm 5 \mathrm{~mm}$ at the channel ends.

by both observations and the nature of high resolution numerical solutions. This scheme is able to reproduce the gross characteristics of the flow, and comparisons with high resolution simulations demonstrate a quite acceptable accuracy. The new scheme is not as accurate as the original procedure [2], where the parametrised model produced results that were only marginally different from those obtained from very high resolution grids. However, the new model covers a much wider dynamical range, and hence is significantly more valuable in modelling the types of flows that are encountered in reality, such as in areas of the GBR. More detailed testing across a range of reef 
gap parameters $(0 \leq w, b \leq 1)$ would be useful, but extremely expensive computationally, and has not yet been attempted.

The scheme has been applied to the computation of flows across large sections of the GBR, for example, in the southern GBR and in Torres Strait. Both are demanding areas for modelling because of the very narrow gaps between the individual reefs and the high pressure gradients that apply. These factors both result in advectively-dominated dynamics, and hence need to use the present scheme to accurately portray the dynamics when reef detail has to be parametrised. Incorporation of the advective terms via the approach described here has led to significantly improved results for tidal models when compared against field data [3]. The present reef parametrisation scheme is not only accurate, but is relatively simple to implement in existing circulation models. It also has other advantages: for example, it is effective in minimising numerical problems that can arise when bathymetry changes rapidly, such as at the edge of a continental shelf. Finally, note that fine-scale realism is not the objective of the present work: rather, the aim is to accurately parametrise the resistance associated with subgrid-scale flow around reefs. Should solutions be required at finer spatial scales, the parametrised model can be used to generate realistic open boundary conditions, which can then be applied to nested models that provide more explicit resolution of spatial detail over limited-area domains.

Acknowledgments: Comments by two anonymous referees are much appreciated. Figure 1 is provided courtesy of NASA/GSFC/LARC/JPL,MISR. ${ }^{1}$

${ }^{1}$ http://www-misr.jpl.nasa.gov/gallery/galhistory/2001_apr_11.html 


\section{References}

[1] L. Bode and L. B. Mason. Application of an implicit hydrodynamic model over a range of spatial scales. In D. Stewart et al., editors, Computational Techniques and Applications: CTAC93, pages 112-121. World Scientific Press, 1994. C1022, C1028

[2] L. Bode, L. B. Mason and J. H. Middleton. Reef parameterisation schemes with applications to tidal modelling. Prog. Oceanogr., 40:285-324, 1997.

http://dx.doi.org/10.1016/S0079-6611(98)00006-8 C1018, C1019, C1021, C1022, C1024, C1028, C1029, C1030, C1031

[3] D. M. Burrage, C. R. Steinberg, L. B. Mason, and L. Bode Tidal corrections for Topex altimetry in the Coral Sea and Great Barrier Reef Lagoon: comparisons with long term tide gauge records.

J. Geophys. Res., 3241, 2003.

http://dx.doi.org/10.1029/2000JC000441 C1018, C1032

[4] T. A. Hardy, L. B. Mason and J. D. McConochie. A wave model for the Great Barrier Reef. Ocean Engng., 28:45-70, 2000. http://dx.doi.org/10.1016/S0029-8018(99)00057-8 C1023

[5] J. J. Dronkers. Tidal computations in rivers and coastal waters. North-Holland, 518 pp., 1964. C1026

[6] S. M. Griffies. Fundamentals of ocean climate models. Princeton University Press, 518 pp., 2004. http://www.pupress.princeton.edu/titles/7797.html C1023

[7] J. M. Huthnance. Flow across reefs or between islands, and effects on shelf-sea motion. Cont. Shelf Res., 4:709-731, 1985. http://dx.doi.org/10.1016/0278-4343(85)90038-X C1020, C1022, C1023 
[8] M. K. James, P. R. Armsworth, L. B. Mason and L. Bode. The structure of reef fish metapopulations: modelling larval dispersal and retention patterns. Proc. Roy. Soc. London, B269:2079-2086, 2002. http://dx.doi.org/10.1098/rspb.2002.2128 C1018

[9] E. J. Metzger and H. E. Hurlburt. Coupled dynamics of the South China Sea, the Sulu Sea, and the Pacific Ocean. J. Geophys. Res., 101:12331-12352, 1996.

http://www .agu.org/pubs/crossref/1996.../95JC03861. shtml C1023

[10] P. J. Roache. Computational fluid dynamics. Hermosa Publishers, 434 pp., 1972. C1028

[11] P. Tkalich, W. C. Pang and P. Sundarambal. Hydrodynamics and eutrophication modeling for Singapore Straits. Proc. 7th workshop on ocean models for the APEC Region, Singapore, pages 5.1-5.9, 2002. C1023

[12] L. J. Waterman. Numerical modelling: building the world ocean. Bull. Aust. Meteorol. Oceanogr. Soc., 11:112-116, 1998. C1023 